\title{
Two New Species of Stenamma (Hymenoptera: Formicidae) from Indian Himalaya With a Revised Key to the Palaearctic and Oriental Species
}

by

\author{
Himender Bharti, Irfan Gul \& Yash Paul Sharma
}

\begin{abstract}
Two new species of genus Stenamma viz Stenamma wilsoni sp. nov. and Stenammajhitingriense sp. nov. are described from Indian Himalaya. This adds two more species to the genus from Indian Himalaya, with only Stenamma kashmirense Baroni Urbani, 1977 described earlier. A revised key to 26 species from Palaearctic and Oriental is provided here with.

Key words: Stenamma, Taxonomy, Myrmicinae, Ants, New species, Key, Indian Himalaya

\section{INTRODUCTION}

Genus Stenamma Westwood 1839 is widely distributed with 47 extant species (Bolton 2011; Liu \& Xu 2011). Recently Liu \& Xu (2011) described three new species of this genus and provided a revised key for the known species of Palaearctic and Oriental regions. Based on molecular evidence Branstetter (2009) redefined Stenamma as monophyletic genus. Earlier DuBois (1998) had revised the Palaearctic and Oriental species of this genus. However, the representation of this genus has been poor from Indian Himalaya, as only one species, Stenamma kashmirense, has been reported by Baroni Urbani (1977). During the course of present study two new species have been recorded and these differ considerably from already described species of this genus. A revised key (modified after Liu \& Xu 2011) which includes 26 known species from Palaearctic and Oriental regions has been provided.
\end{abstract}

\section{MATERIALS AND METHODS}

The specimens were collected by the handpicking method. Digital color images were prepared by Michael Branstetter (California Academy of Sci-

Department of Zoology and Environmental Sciences, Punjabi University, Patiala- 147002, India e-mail: himenderbharti@gmail.com; irfangulhhh@gmail.com; yashraina007@gmail.com 
ences) vide specimen number CASENT 0126224 (Stenamma wilsoni sp. nov.) and CASENT 0126222 (Stenamma jhitingriense sp. nov.). Taxonomic analysis was conducted using a Nikon SMZ 1500 stereo zoom microscope. Morphological terminology for measurements and indices (given in millimeters) includes:

HL-Head length: The length of the head capsule excluding the mandibles, measured in full face view in a straight line from the midpoint of the anterior clypeal margin to the mid-point of the occipital margin.

HW- Head width: The maximum width of the head in full face view, excluding the eyes.

SL-Scapelength: The maximum straight linelength of the scape, excluding the basal constriction or neck that occurs just distal of the condylar bulb.

PW- Pronotal width: The maximum width of the pronotum in dorsal view.

ML- Mesosomal length: The diagonal length of the mesosoma in lateral view from the point at which the pronotum meets the cervical shield to the posterior basal angle of the metapleuron.

ED- Eye diameter: The maximum diameter of the eye.

PL-Petiolelength: Maximum length of petiole, measured from the juncture with propodeum to the juncture with postpetiole.

PH- Petiole height: The perpendicularly maximum height of the petiole, measured from the apex of the node to venter of petiole.

DPW-Dorsal petiole width: Maximum width of petiole, measured across node in dorsal view.

PPL- Postpetiole length: Maximum length of postpetiole, measured from the juncture with petiole to the juncture with gaster.

PPH- Postpetiole height: The perpendicularly maximum height of the postpetiole, measured from the apex of the postpetiolar node to the venter of postpetiole.

PPW- Postpetiole width: Maximum width of postpetiole, measured across the postpetiolar node in dorsal view.

GL: Gaster length: Length of the gaster in lateral view from the anteriormost point of first gastral segment to the posterior-most point.

TL- Total length: HL+ML+PL+PPL+GL.

CI- Cephalic index: HW $\times 100 / \mathrm{HL}$. 
SI- Scape index: SL $\times 100 / H W$.

PI- Petiole index: $\mathrm{PH} \times 100 / \mathrm{PL}$.

PPI- Postpetiole index: PPH $\times 100 /$ PPL.

\section{DESCRIPTION \\ Stenamma wilsoni sp. nov.}

(Figs. 1-3)

Holotype worker: India, Himachal Pradesh, Reckongpeo, 31.540432N, 78.272352E, 2050m above msl, 02.ix.2008, hand picking. Paratypes; 3 workers, same data as holotype (coll. Irfan Gul and Yash Paul Sharma). Depository: PUPAC, Punjabi University Patiala Ant Collection, Patiala, India.

Description of worker (Figs. 1-3):

Worker Measurements: TL 3.58-3.72(3.61); HL 0.78-0.83(0.78) ; HW 0.66-0.71(0.66); SL 0.58-0.62(0.58); PW 0.42-0.46(0.42); ML 0.981.04(0.98); ED 0.07-0.08(0.07); PL 0.40(0.40); PH 0.21-0.22(0.22); DPW 0.14-0.15(0.14); PPL 0.27-0.30(0.27); PPH 0.21-0.22(0.21); PPW 0.210.23(0.21); GL 1.11-1.18(1.18); CI 84.62-86.25(84.62); SI86.96-87.9(87.9); PI 181.82-190.5(190.5); PPI 73.33-77.78(77.78) (4 individuals measured)

Head: Head distinctly rectangular, longer than broad in full face view; occipital margin straight; occipital corners less distinct, moderately round; lateral sides almost parallel; anterior clypeal margin convex, slightly concave in the middle; eyes small, with 5 facets in their greatest diameters, located below the mid points of the lateral sides of head; mandibles triangular, the masticatory border with 3 prominent apical teeth, and 5 less distinct basal teeth; antennae short, 12-segmented, scape stout, falling short by about $1 / 6$ of its length to reach the occipital corners, club 4-segemented.

Mesosoma, Petiole and Postpetiole: In profile view promesonotum high and convex nearly arched, promesonotal suture less distinct;

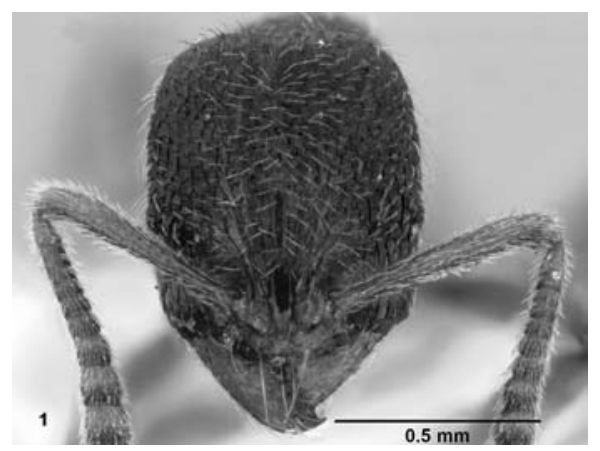

Fig. 1. Stenamma wilsoni sp. nov.; Head, dorsal view. 

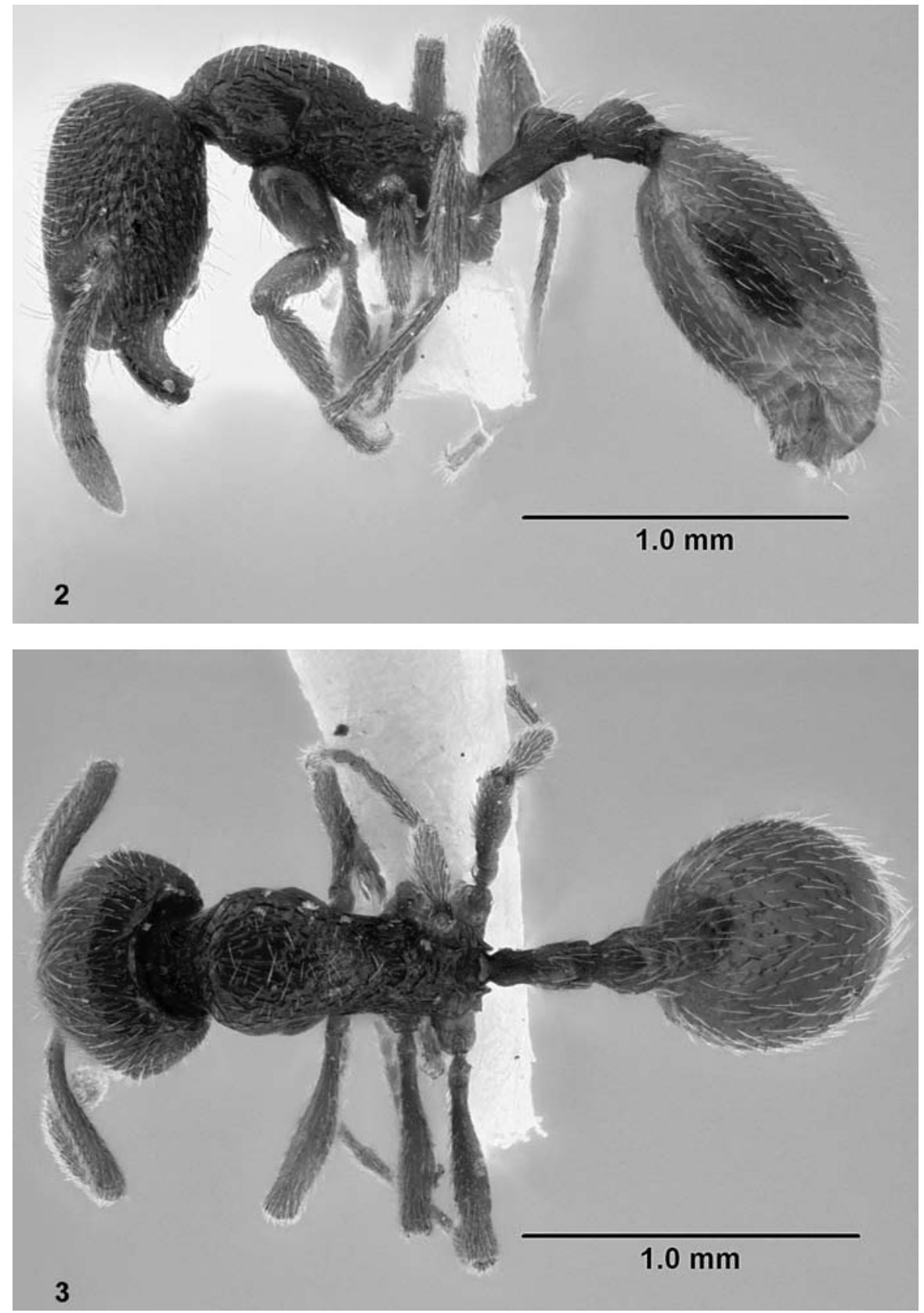

Figs. 2-3. Stenamma wilsoni sp. nov.; 2: Body, lateral view, 3: Body, dorsal view. 
mesometanotum suture well marked making a wide groove; propodeum distinctly lower than promesonotum, convex from side to side, forms a gentle slope towards apex; propodeal spines short, as long as $1 / 3$ the range of their bases; propodeal plates broad, as long as is the length of propodeal spines, posterodorsal corner bluntly angled, posteroventral corner rounded; petiole long, petiolar node approximately as long as anterior peduncle, anteroventral face slightly convex, posterioventral face slightly depressed, anteroventral corner of petiole bluntly angled; postpetiolar dorsum round, ventral face weakly concave, anteroventral corner slightly extruding, tooth like.

Gaster: Gaster ovate, smooth and shining all over.

Sculpture: Head retirugose, except longitudinal irregular rugae below eyes and rugae in between the frontal carinae which run to the occiput; mandibles with less distinct striations; clypeus smooth; promesonotal dorsum rugose, rugae sparse, the central rugaelongitudinal, sides of pronotum with indistinct rugae; propodeum and sides of mesonotum retirugose; propodeal declivity smooth; dorsum of petiolar peduncle smooth without longitudinal carina; petiole and postpetiole interweaved with fine, less distinct longitudinal rugae; gaster smooth and shining except a few short rugae at base.

Pilosity: Body clothed with suberect to erect hairs, more abundant on head and gaster; on mesosomal surface hairs erect, sparse and scattered; shorter subdecumbent hairs on antennae and legs; decumbent pubescence on antennae and legs, more dense on antennal funiculus.

Color: Head, mesosoma, petiole, postpetiole and middle of gaster reddish brown; mandibles, antennae, legs, and remaining part of gaster yellowish brown; eyes black; pilosity yellowish white.

Distribution and Habitat: The species has been collected from a single locality of North-West region of Indian Himalaya. The specimen has been collected by hand picking, from a rotten log of wood, in open woodland on a hill slope.

Etymology: The species is named in the honour of Prof. E. O. Wilson.

Remarks: The species is significantly different from already described species of this genus. The species has a very distinct sculpture of head and mesosoma, differs in shape and size of propodeal plates, in propodeal spines and petiole. Due to petiolar node as long as its anterior peduncle and scape not reaching to the occipital corners, this species can be easily separated from 
Stenamma kashmirense Baroni Urbani, 1977 (the only species reported from Indian Himalaya hitherto). However Stenamma wilsoni sp. nov. is somewhat allied to Stenammajeriorum DuBois, 1998 and Stenamma lippulum Nylander, 1849 , but can be easily separated from former by parallel sides of head and propodeal spines as long as are the propodeal plates, where as lateral sides of head are strongly convex in Stenamma jeriorum and propodeal spines shorter than propodeal plates. From Stenamma lippulum this new species is considerably distinct on the basis of the sculpture of head, where the rugae make concentric loop like structures above eyes, whereas in this new species sculpture of head is retirugose.

\section{Stenamma jhitingriense sp. nov. \\ (Figs 4-6)}

Holotype worker: India, Himachal Pradesh, Jhitingri, 32.006475N, 76.839237E, 1750m above msl,27.vi.2010, hand picking. No paratypes. (coll. Yash Paul Sharma and Irfan Gul). Depository: PUPAC, Punjabi University Patiala Ant Collection, Patiala, India.

Description of worker (Figs. 4-6):

Worker Measurements: TL (3.44); HL (0.77); HW (0.66); SL (0.57); PW (0.43); ML (1.0); ED (0.07); PL (0.40); PH (0.20); DPW (0.15); PPL (0.29); PPH (0.21); PPW (0.21); GL (0.98); CI (85.71); SI (86.36); PI (200); PPI (72.41) (1individual measured).

Head: Head rectangular, longer than broad in full face view; occipital margin straight; occipital corners less distinct, more round; lateral sides almost parallel; anterior clypeal margin convex, emarginate in the middle; eyes small, with 4 less distinct facets in their greatest diameters, located below the mid points of the lateral sides of head; mandibles triangular, the masticatoryborderwith 3 prominent apical

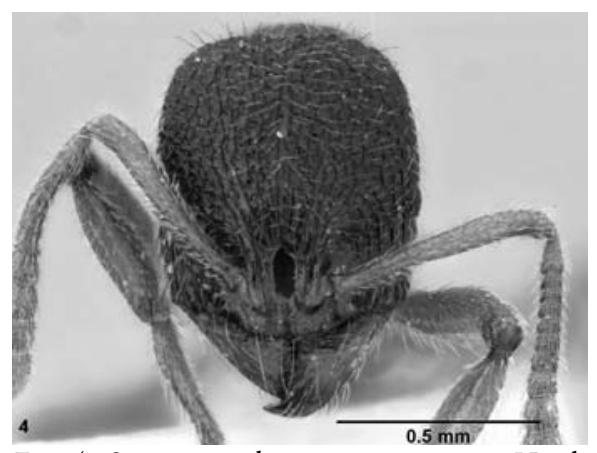

Fig. 4. Stenamma jhitingriense sp. nov.; Head, dorsal view. 

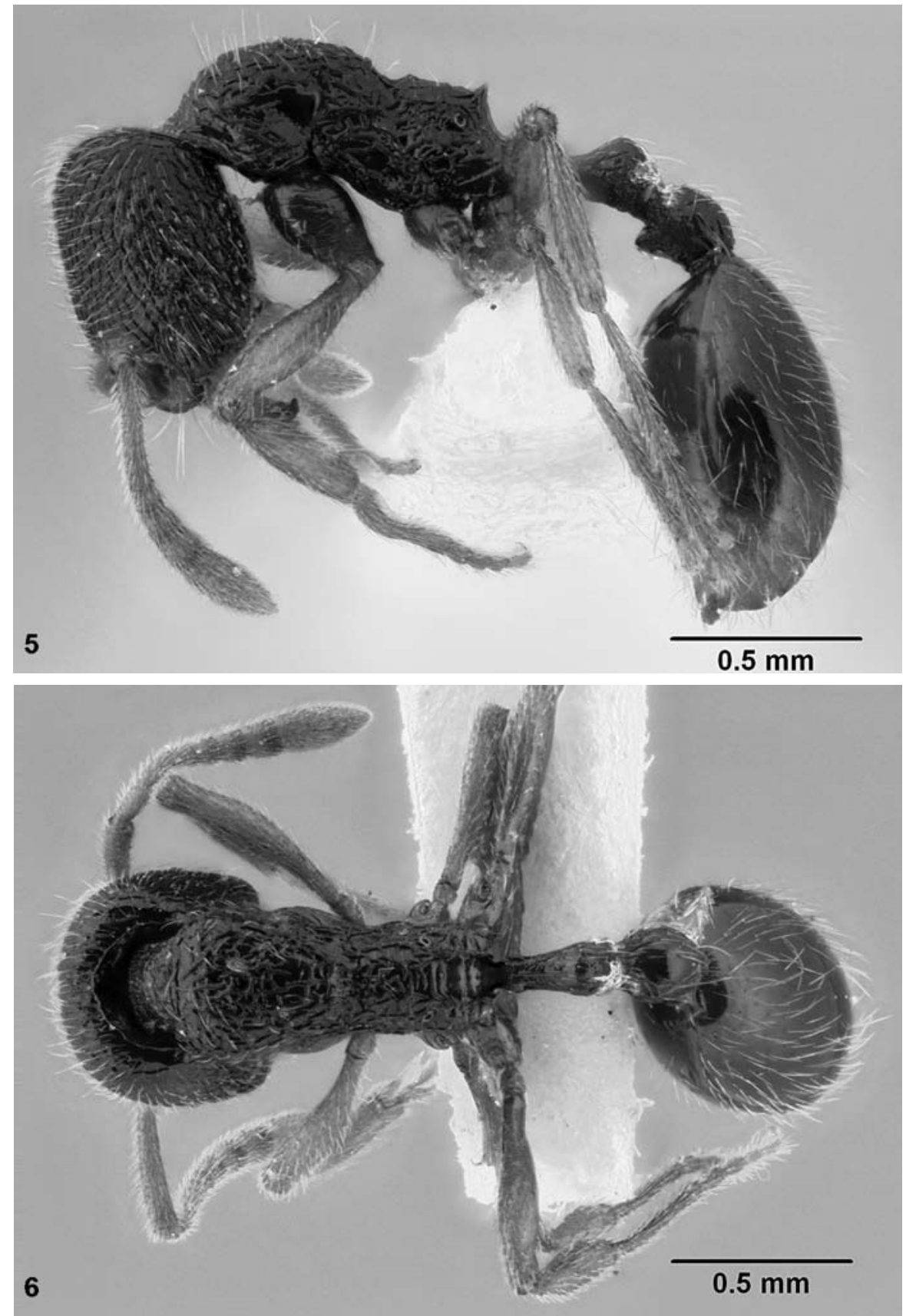

Figs. 5-6. Stenamma jhitingriense sp. nov.; 5: Body, lateral view, 6: Body, dorsal view. 
teeth, and 5 less distinct basal teeth; antennae short, 12-segmented, scape stout, falling short by nearly $1 / 6$ of its length to reach the occipital corners, club 4-segmented.

Mesosoma, Petiole and Postpetiole: In profile view promesonotum high and convex nearly arched, promesonotal sutureless distinct; mesometanotum suture well marked making a deep and wide groove; propodeum distinctly lower than promesonotum, dorsum more flat, forms a gentle slope towards apex; propodeal spines short, acute, as long as $2 / 5$ the range of their bases; propodeal plates broad, roughly rectangular, slightly shorter than propodeal spines, bluntly angled on posterodorsal and posteroventral corners; petiole long, petiolar node approximately as long as anterior peduncle, anteroventral face straight, posteroventral face slightly concave, anteroventral corner of petiole bluntly angled; postpetiolar dorsum round, ventral face weakly concave, anteroventral corner strongly extruding.

Gaster: Gaster ovate, smooth and shining all over.

Sculpture: Head retirugose, except longitudinal irregular rugae in between the frontal carinae; mandibles indistinctly striate; clypeus smooth; promesonotal dorsum with distinct retirugose sculpture, sides of pronotum with indistinct rugae; propodeum and sides of mesonotum retirugose; propodeal declivity smooth; dorsum of petiolar peduncle with a finelongitudinal central carina; petiole and postpetiole interweaved with fine, less distinct longitudinal rugae; gaster smooth and shining except a few short rugae at base.

Pilosity: Body clothed with sub-erect to erect hairs, more abundant on the head and gaster; on mesosomal surface hairs erect, sparse and scattered; shorter subdecumbent hairs on antennae and legs; decumbent pubescence on antennae and legs, more dense on antennal funiculus.

Color: Head, mesosoma, petiole, postpetiole and middle of gaster blackish brown; mandibles, antennae, legs, and remaining part of gaster yellowish brown; eyes black; pilosity yellowish white.

Distribution and Habitat: The specimen has been collected from a forest area of Jhitingri, Himachal Pradesh in North-West Himalayas. The specimen has been collected from leaf litter by handpicking.

Etymology: The species is named after the type locality, Jhitingri.

Remarks: Stenamma jhitingriense sp. nov. is significantly different from previously reported species of this genus due to the following combination 
of characters: blunt anteroventral corner of petiole, scape not reaching occipital corners, equal length of petiole and peduncle, longer propodeal spines than propodeal plates and blackish brown color of body. However it shows few affinities with Stenamma gurkbale DuBois, 1998, Stenamma punctiventre Emery, 1908 and Stenamma koreanense Lyu, DuBois \& Cho, 2002. In Stenamma gurkhale and Stenamma koreanense the petiolar anteroventral corner is acutely toothed, Stenamma punctiventre has an anteroventral corner of petiole extended and finger-like, but in Stenamma jhitingriense the anteroventral corner of the petiole is blunt. It is easily separated from Stenamma kashmirense Baroni Urbani, 1977, as in Stenamma kashmirense the peduncle is less than the half the length of petiole, and the scape reaches the occipital corners distinctly.

\section{A REVISED KEY TO THE KNOWN PALAEARCTIC AND ORIENTAL SPECIES OF STENAMMA BASED ON THE WORKER CASTE (MODIFIED AFTER LIU \& XU 2011)}

1. In full face view, antennal scapes distinctly surpassed occipital corners.. 2

- In full face view, antennal scapes reached to or not reached to occipital corners .....................................................................................................

2. In profile view, petiolar node distinctly shorter than anterior peduncle .....3

- In profile view, petiolar node as long as or longer than anterior peduncle

3. In full face view, occipital corners rounded. In profile view, metanotal groove deeply depressed. Propodeal plates narrow, nearly triangular (DuBois, 1998: figs. 200-202). (Distribution: Japan)

S. nipponense Yasumatsu \& Murakami

- In full face view, occipital corners roundly prominent. In profile view, metanotal groove shallowly depressed. Propodeal plates broad, nearly trapezoid (Figs. 1-3). (Distribution: China (Yunnan Province))

S. ailaoense Liu \& Xu

4. In full face view, occipital margin roundly convex ………………………...

- In full face view, occipital margin nearly straight ............................................ 6

5. In profile view, dorsum of promesonotum evenly convex. Propodeal dorsum straight. Propodeal plates nearly trapezoid, truncated at apices (DuBois, 1998: figs. 187-190). (Distribution: Algeria, Morocco, Tunisia) 
S. msilanum Forel

- In profile view, dorsum of promesonotum nearly straight. Propodeal dorsum weakly convex. Propodeal plates nearly semicircular, rounded at apices (DuBois, 1998: figs. 240-242). (Distribution: Southern Europe, mostly France and Italy) S. petiolatum Emery

6. In full face view, head broadest at back, narrowed forward, lateral sides relatively straight

- In full face view, head broadest in the middle, lateral sides evenly convex

7. Mandibles with 7 teeth. In profile view, anteroventral corner of petiole extruding and forming a rightly angled tooth (DuBois, 1998: figs. 328330). (Distribution: England, Belgium) ............... . westwoodii Westwood

- Mandibles with 9 teeth. In profile view, anteroventral corner of petiole weakly convex, not forming a tooth (DuBois, 1998: figs. 184-186). (Distribution:Russia)

S. lippulum Nylander

8. In profile view, dorsum of promesonotum evenly convex. Dorsum of petiolar node roundly prominent (DuBois, 1998: figs. 280-282). (Distribution:Spain) ........................................................ sardoum Emery

- In profile view, dorsum of promesonotum nearly straight. Dorsum of petiolar node rounded (DuBois, 1998: figs. 277-279). (Distribution: Sardinia)..

S. sardoum Emery

9. In full face view, antennal scapes distinctly not reached to occipital corners

- In full face view, antennal scapes reached to occipital corners................. 16

10. In profile view, petiolar node distinctly longer than anterior peduncle (Dubois, 1998: figs. 310-314). (Distribution: Eastern Russia)

S. ussuriense Arnol'di

- In profile view, petiolar node approximately as long as anterior peduncle

11. In profile view, anteroventral corner of petiole extended and finger-like (DuBois, 1998: figs. 269-271). (Distribution: Morocco)

S. punctiventre Emery

- In profile view, anteroventral corner of petiole prominent, tooth-like or bluntly angled

12. In profile view, anteroventral corner of petiole acutely toothed (Lyu etal., 
2002: figs. 1-4). (Distribution: Korea)

S. koreanense Lyu, DuBois \& Cho

- In profile view, anteroventral corner of petiole bluntly angled ................ 13

13. In full face view, lateral sides of head strongly convex. In profile view, propodeal spines shorter than propodeal plates (DuBois, 1998: figs. 148152). (Distribution: Pakistan)........................................ S. jeriorum DuBois

- In full face view, lateral sides of head weakly convex or parallel. In profile view, propodeal spines as long as or longer than propodeal plates ........ 14

14. In full face view, lateral sides of head weakly convex. In profile view, propodeal spines as long as or longer than propodeal plates. Head dorsum above eyes with rugae forming concentric loop like structures (DuBois, 1998: figs. 170-177). (Distribution: Azerbaijan, Georgia, southern Russia) ......

S. lippulum Nylander

- In full face view, lateral sides of head parallel. In profile view, propodeal spines longer or equal to propodeal plates. Head dorsum above eyes with rugae not forming loop like structures. (Distribution: Himalaya)........ 15

15. Dorsum of petiolar peduncle smooth without a central longitudinal carina. Postpetiolar anteroventral corner slightly extruding, tooth like. Propodeal plates broad, as long as is the length of propodeal spines

S. wilsoni sp. nov.

- Dorsum of petiolar peduncle with a central longitudinal carina. Postpetiolar anteroventral corner strongly extruding. Propodeal plates broad, roughly rectangular, slightly shorter than propodeal spines

S. jhitingriense sp. nov.

16. In profile view, petiolar node distinctly shorter than anterior peduncle. 17

- In profile view, petiolar node as long as or longer than anterior peduncle 18

17. Eyes with 8-9 ommatidia in the maximum diameter. Mesopleura retirugose (DuBois, 1998: figs. 226-228). (Distribution:Japan, China (Sichuan Province) .......................................................................... S. owstoni Wheeler

- Eyes with 4 ommatidia in the maximum diameter. Mesopleura longitudinally rugose (Figs. 4-6 ). (Distribution: China (Yunnan Province))........... S. wumengense Liu \& $\mathrm{Xu}$

18. In profile view, petiolar node distinctly longer than anterior peduncle .. 
- In profile view, petiolar node about as long as anterior peduncle............ 21

19. In profile view, propodeal spines longer than propodeal plates. Propodeal plates triangular, bluntly angled at apices (DuBois, 1998: figs. 191-193). (Distribution: Algeria, Morocco, Tunisia) S. msilanum Forel

- In profile view, propodeal spines shorter than propodeal plates. Propodeal plates nearly trapezoid, truncated at apices ............................................... 20

20. In profile view, dorsum of promesonotum roundly convex. Propodeal spines posteriorly curved. Dorsum of petiolar node narrowly prominent (DuBois, 1998: figs. 131-135). (Distribution: Tajikstan)

S. hissarianum Arnol'di

- In profile view, dorsum of promesonotum relatively straight. Propodeal spines not posteriorly curved. Dorsum of petiolar node broadly rounded (DuBois, 1998: figs. 251-255). (Distribution: Kazakhstan, Kirghizia) ..

S. picetojuglandeti Arnol'di

21. In profile view, anteroventral corner of petiole acutely toothed. Petiolar node lower, with dorsum rounded (DuBois, 1998: figs. 126-129). (Distribution: Nepal)

S. gurkhale DuBois

- In profile view, anteroventral corner of petiole bluntly angled or roundly prominent. Petiolar node higher, with dorsum roundly or narrowly promi-

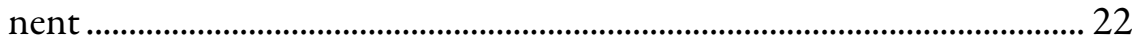

22. In full face view, head distinctly narrowed forward ................................ 23

- In full face view, head not distinctly narrowed forward ......................... 25

23. In full face view, occipital margin weakly convex. In profile view, dorsum of petiolar node broadly rounded, both anterior and posterior faces convex (DuBois, 1998: figs. 290-295). (Distribution: From Spain to Turkey) ... S. striatulum Emery

- In full face view, occipital margin straight. In profile view, dorsum of petiolar node narrowly prominent, both anterior and posterior faces relatively straight

24. In full face view, occipital corners roundly prominent. In profile view, dorsum of promesonotum weakly convex (DuBois, 1998: figs. 237-239). (Distribution: Southern Europe, mostly France and Italy)

S. petiolatum Emery

- In full face view, occipital corners rounded. In profile view, dorsum of 
promesonotum strongly convex (DuBois, 1998: figs. 111-119). (Distribution: Georgia, Southern Russia)

S. georgii Arnol'di

25. In full face view, occipital margin evenly roundly convex (DuBois, 1998: figs. 100-102). (Distribution: Throughout Europe) ..S. debile (Foerster)

- In full face view, occipital margin straight or weakly concave in the middle 26

26. In profile view, propodeal dorsum nearly horizontal............................... 27

- In profile view, propodeal dorsum slope down backward .........................2 28

27. In profile view, dorsum of promesonotum roundly convex. Propodeal dorsum weakly convex (DuBois, 1998: figs. 214-217). (Distribution: France (Corsica), Italy (Sardinia), Spain) ..S. orousseti Casevitz-Weulersse

- In profile view dorsum of promesonotum nearly straight. Propodealdorsum straight (DuBois, 1998: figs. 163-168). (Distribution: Russia (Kuril)...

S. kurilense Arnol'di

28. In profile view, propodeal declivity roundly concave. Anterior face of petiolar node formed a strong depression with peduncle (DuBois, 1998: figs. 284-288). (Distribution: Uzebekistan)............ S. sogdianum Arnol'di

- In profile view, propodeal declivity straight. Anterior face of petiolar node formed a weak depression with peduncle

29. In profile view, propodeal spines shorter than propodeal plates. Propodeal plates truncated at apices (DuBois, 1998: figs. 154-161). (Distribution: India (Kashimir), Pakistan) ........................ S. kashmirense Baroni Urbani

- In profile view, propodeal spines as long as propodeal plates. Propodeal plates rounded at apices (Figs. 7-9). (Distribution: China (Tibet, Sichuan Province). S. yaluzangbum Liu \& $\mathrm{Xu}$

\section{ACKNOWLEDGMENTS}

Sincere thanks to Michael Branstetter (California Academy ofSciences) for getting this species digitized. Financial assistance rendered by Department of Science and Technology (Grant No. SR/SO/AS-65/2007), Govt. of India, New Delhi is gratefully acknowledged.

\section{REFERENCES}

Baroni Urbani, C. 1977. Ergebnisse der Bhutan-Expedition 1972 des Naturhiswrischen Museums in Basel. Hymenoptera: Fam. Formicidae. Genus Stenamma, con una nuova species del Kashmir. Enwmologica Basiliensia 2: 415-422. 
Bolton, B. 2011. Bolton's Catalogue and Synopsis, in http://gap.entclub.org/ "Version: 3 January 2011.

Branstetter, M.G. 2009. The ant genus Stenamma Westwood (Hymenoptera: Formicidae) redefined, with a description of a new genus Propodilobus. Zootaxa 2221: 41-57.

DuBois, M. B. 1998. A revision of the ant genus Stenamma in the Palaearctic and Oriental regions. Sociobiology 29: 193-4.03.

Liu, X. \& Z.H. Xu 2011. Three New Species of the Ant Genus Stenamma (Hymenoptera: Formicidae) from Himalaya and the Hengduan Mountains With a Revised Key to the Known Species of the Palaearctic and Oriental Regions. Sociobiology 58: 733747.

Westwood, J.O. 1839. An Introduction to the Modern Classification of insects; founded on the natural habits and corresponding organisation of the different families 2 (part 11), pp. 193-224. London.

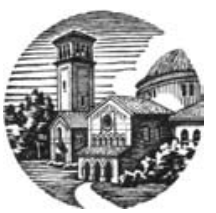

